\title{
Point-of-care ultrasonography for the diagnosis of small bowel obstruction in the emergency department
}

\author{
Joshua Guttman, MD*; Michael B. Stone, MD; Heidi H. Kimberly, MD'; \\ Joshua S. Rempell, MD, $\mathrm{MPH}^{\dagger}$
}

\section{ABSTRACT}

Small bowel obstruction (SBO) is a common cause of acute abdominal pain presenting to the emergency department (ED). Although the literature is limited, point-of-care ultrasonography (POCUS) has been found to have superior diagnostic accuracy for SBO compared to plain radiography; however, it is rarely used in North America for this. We present the case of a middle-aged man who presented with abdominal pain where POCUS by the emergency physician early in the hospital course expedited the diagnosis of SBO and led to earlier surgical consultation. The application of POCUS for SBO is easily learned and applied in the ED. POCUS for SBO may obviate the need for plain radiography and expedite patient care.

\section{RÉSUMÉ}

L'occlusion de l'intestin grêle (OIG) est une cause fréquente de douleur abdominale aiguë au service des urgences (SU). Malgré une documentation peu abondante, l'échographie au point de service (EPS) se révèle supérieure à la radiographie simple quant à l'exactitude diagnostique dans I'OIG; toutefois, l'emploi de l'EPS dans ce contexte est peu étendu en Amérique du Nord. Sera exposé dans I'article le cas d'un homme d'âge mûr, qui a consulté pour des douleurs abdominales; le recours à l'EPS par l'urgentologue peu de temps après l'arrivée à l'hôpital a permis d'accélérer la pose du diagnostic d'OIG et de demander rapidement une consultation en chirurgie. L'application de I'EPS dans I'OIG s'apprend et s'applique facilement au SU. L'EPS dans le contexte de I'OIG peut ainsi rendre obsolète la radiographie simple et accélérer les soins aux patients.

Keywords: emergency ultrasonography, point-of-care ultrasonography, small bowel obstruction
Small bowel obstruction (SBO) is a common presentation to the emergency department (ED) and represents $15 \%$ of hospital admissions for acute abdominal complaints. In the early stages of the disease, the diagnosis remains a challenge as signs and symptoms overlap with other entities. ${ }^{1}$ Plain radiography, although traditionally recommended as the initial diagnostic imaging modality of choice, ${ }^{2}$ has a sensitivity of only 59 to $77 \% .^{2,3}$ When clinical and radiographic assessment is indeterminate, computed tomography (CT) becomes the test of choice due to its superior resolution and increased ability to identify both obstruction and its etiology. ${ }^{2}$ However CT scanning is expensive, confers long-term risks associated with radiation, and is not available 24 hours a day at many centres. In recent years, point-of-care ultrasonography (POCUS) has been described as a potential imaging modality for the diagnosis of SBO. ${ }^{4-6} \mathrm{We}$ present the case of a middleaged man who presented with abdominal pain where POCUS by the emergency physician early in the hospital course expedited the diagnosis of SBO and led to earlier surgical consultation and directed therapy. The application of POCUS for SBO in the ED, and its potential to obviate the need for plain radiography and expedite patient care, is subsequently discussed.

\section{CASE REPORT}

A 57-year-old man with a history of sigmoid colectomy for rectal adenocarcinoma and previous partial small

From the *Emergency Medicine Residency Program, McGill University Health Centre, Montreal, QC, and tDivision of Emergency Ultrasound, Department of Emergency Medicine, Brigham and Women's Hospital, Boston, MA.

Correspondence to: Dr. Joshua Guttman, Emergency Medicine Residency Program, McGill University Health Centre, 687 Pine Avenue West, Room A4.62, Montreal, OC H3A 1A1; Joshua.guttman@mail.mcgill.ca.

This article has been peer reviewed.

(c) Canadian Association of Emergency Physicians 
bowel obstruction presented to the ED complaining of abdominal pain, distention, nausea and vomiting, and decreased stool output. On examination, his heart rate was 109 beats per minute, and his blood pressure was 103/71 mm Hg. He was afebrile and had a normal oxygen saturation. His physical examination was notable for significant abdominal distention and diffuse tenderness without rebound or guarding. Immediate bedside abdominal ultrasonography was performed by the emergency physician and revealed multiple fluidfilled dilated loops of bowel with bowel wall thickening consistent with SBO (Figure 1). Based on the ultrasound findings, the surgical service was immediately involved and was expediently admitted to the surgical ward. Subsequent abdominal plain radiography revealed moderate distention of the colonic and small bowel loops (Figure 2). Abdominal CT scanning was performed and revealed marked distention of the distal small bowel loops and entire colon (Figure 3).

\section{DISCUSSION}

The use of ultrasonography by radiologists as the initial imaging study in the assessment of possible SBO in patients presenting with abdominal pain is increasingly common in Europe. However, ultrasonography is uncommonly used by radiologists for this indication in North America..$^{7-9}$ When performed by radiologists, ultrasonography has a sensitivity of $95 \%$ and a specificity of $84 \%$ for SBO. ${ }^{10}$ Radiologists use several measures when assessing for SBO by ultrasonography: dilated bowel $(>25 \mathrm{~mm}$ at the jejunum and $>15 \mathrm{~mm}$ at the ileum) over the length of three bowel loops, increased bowel wall thickness $(>2 \mathrm{~mm}$ ), increased peristalsis, increased intestinal content (fluid and echoes), and the presence of extraluminal fluid. ${ }^{7,10}$

In the ED, POCUS may be of particular value in evaluating patients with possible $\mathrm{SBO}$ as it can be performed quickly, is inexpensive, and has no associated radiation. POCUS for SBO has the potential to obviate the need for plain radiography and expedite surgical consultation and treatment prior to obtaining a CT scan.

Two studies have evaluated the diagnostic accuracy of POCUS by emergency providers in the assessment of SBO. Unluer and colleagues prospectively enrolled 174 adults presenting to the ED with suspected SBO. Trained emergency medicine (EM) residents performed the ultrasonography. A positive sonogram was defined as one with the presence of two or all of the following: 1) dilated small bowel loops in three segments, 2) increased peristalsis, and 3) a collapsed colonic lumen. A sensitivity of $97.7 \%$ and a specificity of $92.7 \%$ were reported. The gold standard for the presence of SBO was surgical pathology and for the absence of SBO was negative surgical findings (if surgery was done), negative CT (if obtained), or a benign clinical course as determined on telephone follow-up at 1 month. Of the three criteria, dilated bowel loops had the best diagnostic accuracy, with a sensitivity of $94.2 \%$ and a specificity of $93.8 \%$.

Jang and colleagues prospectively enrolled adults presenting to the ED who were undergoing CT scanning for suspected SBO. ${ }^{6}$ Ultrasonography was performed by trained EM residents. A positive sonogram was defined as having one of the following two criteria: 1) fluid-filled, dilated bowel loops (>25 mm) proximal to collapsed or normal bowel or 2) decreased
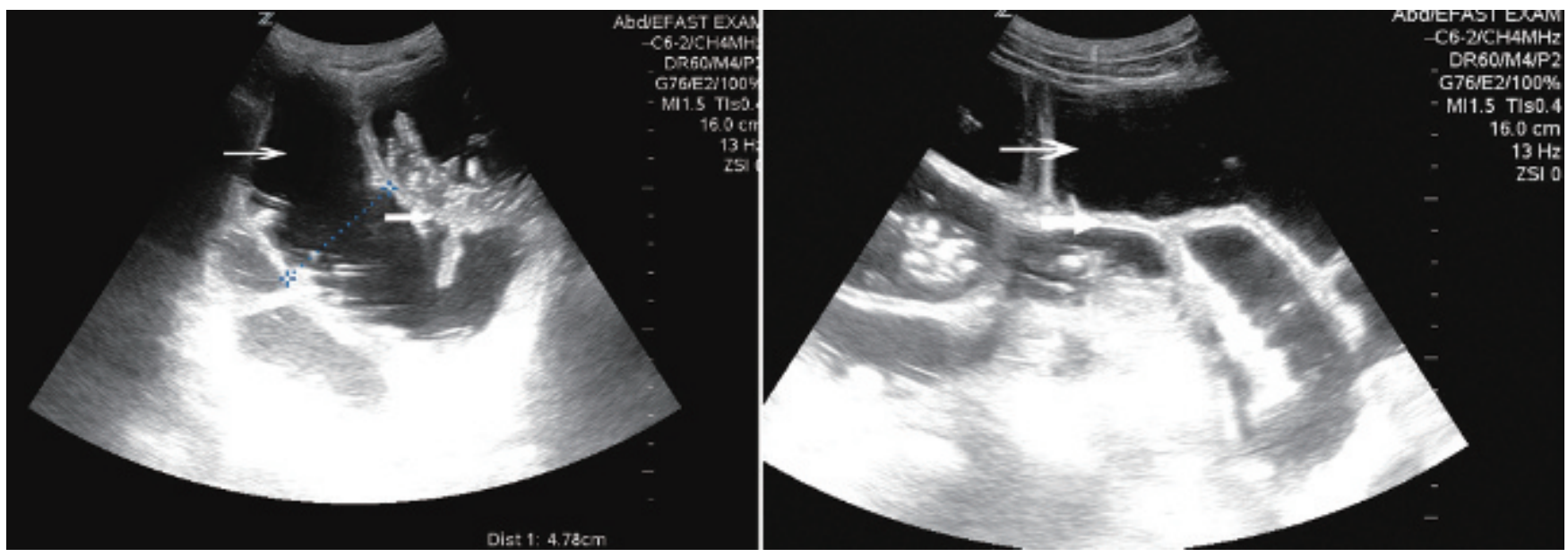

Figure 1. Sonograms showing dilated loops of bowel (thin arrow) and thickened bowel wall (large arrow). 


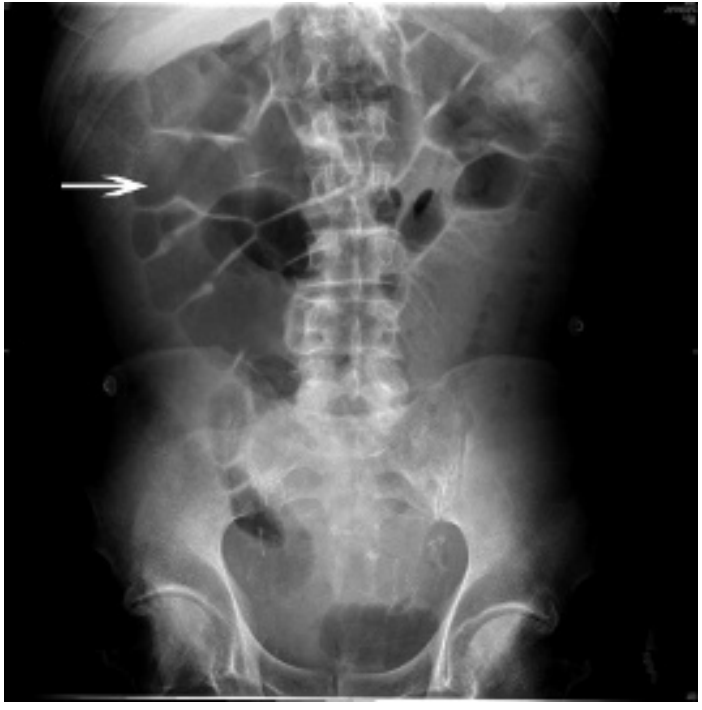

Figure 2. Plain film of patient showing dilated loops of bowel (arrow).

peristalsis. Overall, they found a sensitivity of $93.9 \%$ and a specificity of $81.4 \%$ compared to CT scan. Dilated bowel alone had a sensitivity of $90.9 \%$ and a specificity of $83.7 \%$. Decreased peristalsis alone had a sensitivity of $27.3 \%$ and a specificity of $97.7 \%$. The low sensitivity and high specificity of decreased peristalsis alone are not surprising as decreased peristalsis has been described as a late finding, seen in the setting of strangulation. In both the aforementioned studies, the diagnostic accuracy of ultrasonography was found to be superior to plain radiography. The generalizability of these studies may be limited by the high prevalence of SBO in the study populations $(43 \%$ in the Jang and

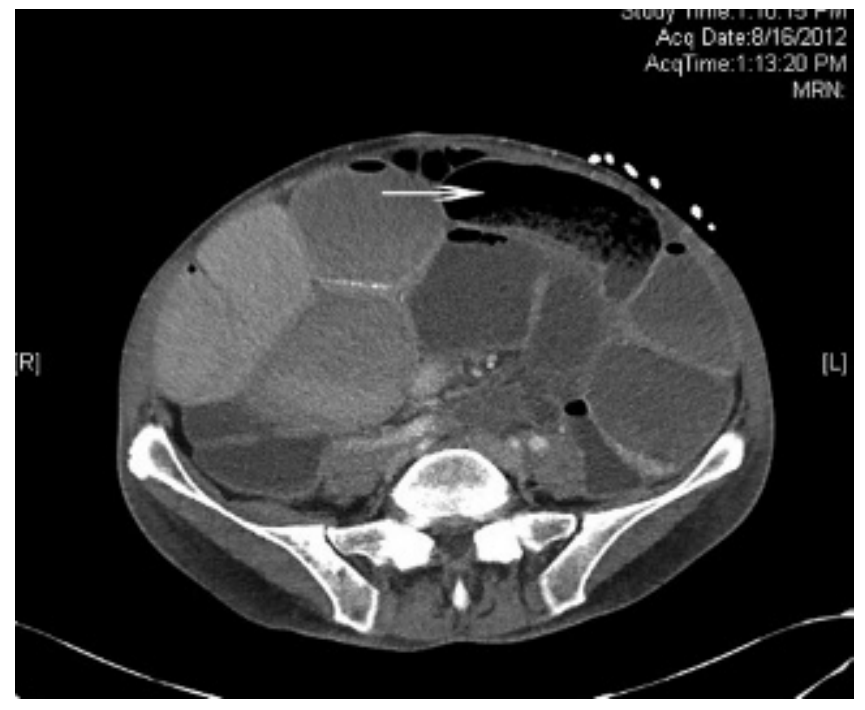

Figure 3. Cross-sectional image of a computed tomographic scan showing dilated loops of bowel (arrow). colleagues study ${ }^{6}$ and $48 \%$ in the Unluer and colleagues study ${ }^{5}$.

POCUS has the potential to be used to detect complications of SBO. Chen and colleagues prospectively compared the diagnostic accuracy of POCUS and plain radiography for the diagnosis of pneumoperitoneum. ${ }^{11}$ Ultrasonography was found to have a higher sensitivity (93\%) than plain radiography $(79 \%)$ against a gold standard of laparotomy findings or, if no laparotomy was performed, against endoscopy or CT scan findings. The specificity of both modalities was identical at $64 \%$.

Evaluating an ED patient with potential SBO using POCUS has several advantages. POCUS may obviate the need for plain radiography by accurately assessing both evidence of SBO and the presence or absence of pneumoperitoneum. In making a diagnosis immediately at the bedside, the emergency physician can expedite surgical consultation and disposition. The delay to CT scan, whether for CT availability or for oral contrast administration, can be a matter of hours. In situations where plain radiography would have been indeterminate, a sonogram proving the presence of SBO may allow patients to be admitted to the surgical ward to await a CT scan, expediting ED flow. POCUS may also lead to earlier treatment, specifically nasogastric tube suction and analgesic administration.

As with any ultrasound application, the diagnostic accuracy of POCUS for SBO is dependent on the ultrasonographer's training and experience. In the Unluer and colleagues study, EM residents performing the ultrasonography had 3 hours of didactic education sessions and 3 hours of hands-on training. ${ }^{5}$ In the Jang and colleagues study, EM residents who had taken a basic introductory POCUS course underwent only 10 minutes of training and five practice scans before enrolling patients. ${ }^{6}$ Given the minimal additional training required, this application of EM POCUS seems feasible.

The utility of POCUS in undifferentiated abdominal pain requires further study. Although both the Unluer and colleagues and Jang and colleagues studies suggest that emergency physicians have excellent diagnostic accuracy with respect to detecting SBO, 5,6 these results may not be generalizable elsewhere. POCUS has no associated risks and may provide immediate evidence of obstruction and thus expedite patient care. Future research should confirm the diagnostic accuracy of POCUS for SBO in a more 
undifferentiated patient population with a lower disease prevalence than the two reported studies. An important question to address is whether POCUS performed early in the care of patients with undifferentiated abdominal pain improves outcomes. The effect, if any, of POCUS on time to consultation, treatment, ED length of stay, and use of plain radiography in patients with SBO or undifferentiated abdominal pain is also important to determine.

\section{CONCLUSION}

We present the case of a middle-aged man who presented with abdominal pain where POCUS by the emergency physician early in the hospital course expedited the diagnosis of SBO and led to earlier surgical consultation. Emergency physician-performed POCUS to detect SBO may have a diagnostic accuracy superior to plain radiography and is easily learned and performed. Although studies confirming improved patient outcomes or quality of care are required, POCUS for suspected SBO has the potential to quickly narrow the differential diagnosis and expedite therapeutic interventions, surgical consultations, and patient disposition.

Competing interests: None declared.

\section{REFERENCES}

1. Torrey S, Henneman P. Disorders of the small intestine. In: Marx JA, editor. Rosen's emergency medicine: concepts and clinical practice. 7th ed. Philadelphia: Elsevier; 2010. p. 1184-92.
2. Maung AA, Johnson DC, Piper GL, et al. Evaluation and management of small-bowel obstruction: an Eastern Association for the Surgery of Trauma practice management guideline. 7 Trauma Acute Care Surg 2012;73(5 Suppl 4):S362-9, doi:10.1097/TA.0b013e31827019de.

3. Suri S, Gupta S, Sudhakar PJ, et al. Comparative evaluation of plain films, ultrasound and CT in the diagnosis of intestinal obstruction. Acta Radiol 1999;40:422-8, doi:10. 3109/02841859909177758.

4. Ogata M, Mateer JR, Condon RE. Prospective evaluation of abdominal sonography for the diagnosis of bowel obstruction. Ann Surg 1996;223:237-41, doi:10.1097/00000658199603000-00002.

5. Unluer EE, Yavasi O, Eroglu O, et al. Ultrasonography by emergency medicine and radiology residents for the diagnosis of small bowel obstruction. Eur 7 Emerg Med 2010;17:260-4, doi:10.1097/MEJ.0b013e328336c736.

6. Jang TB, Schindler D, Kaji AH. Bedside ultrasonography for the detection of small bowel obstruction in the emergency department. Emerg Med 7 2011;28:676-8, doi:10.1136/emj. 2010.095729.

7. Grassi R, Romano S, D'Amario F, et al. The relevance of free fluid between intestinal loops detected by sonography in the clinical assessment of small bowel obstruction in adults. Eur 7 Radiol 2004;50:5-14, doi:10.1016/j.ejrad.2003.11.009.

8. Ko YT, Lim JH, Lee DH, et al. Small bowel obstruction: sonographic evaluation. Radiology 1993;188:649-53.

9. Czechowski J. Conventional radiography and ultrasonography in the diagnosis of small bowel obstruction and strangulation. Acta Radiol 1996;37:186-9, doi:10.3109/ 02841859609173442 .

10. Schmutz GR, Benko A, Fournier L, et al. Small bowel obstruction: role and contribution of sonography. Eur Radiol 1997;7:1054-8, doi:10.1007/s003300050251.

11. Chen SC, Wang HP, Chen WJ, et al. Selective use of ultrasonography for the detection of pneumoperitoneum. Acad Emerg Med 2002;9:643-5, doi:10.1111/j.1553-2712. 2002.tb02307.x. 